\title{
Head and neck cancer patient experience of a new dietitian-delivered health behaviour intervention: 'you know you have to eat to survive'
}

\author{
Kristen McCarter ${ }^{1}$ (D) Amanda L. Baker ${ }^{1} \cdot$ Ben Britton $^{1} \cdot$ Sean A. Halpin ${ }^{2} \cdot$ Alison Beck $^{1} \cdot$ Gregory Carter $^{1}$. \\ Chris Wratten ${ }^{3}$ - Judith Bauer ${ }^{4} \cdot$ Luke Wolfenden $^{1} \cdot$ Katrina Burchell $^{1} \cdot$ Erin Forbes $^{1}$
}

Received: 10 July 2017 / Accepted: 19 December 2017 / Published online: 27 January 2018

(C) The Author(s) 2018. This article is an open access publication

\begin{abstract}
Purpose The aim of this study was to explore head and neck cancer (HNC) patient experiences of a novel dietitian delivered health behaviour intervention.

Methods This study is a qualitative study which employed semi-structured individual interviews using open and axial coding and then final selective coding to organise the data. Patients with $\mathrm{HNC}$ who had participated in a dietitian delivered health behaviour intervention to reduce malnutrition were invited to discuss their experience of this intervention. Individual interviews were conducted, transcribed and analysed using grounded theory.

Results Nine patients participated in the interviews. Four dimensions were identified in the initial coding process: 'information', which described patients' desire for tailored advice during their treatment; 'challenges of treatment experience', which described the difficulties related to treatment side effects; 'key messages: importance of eating and maintaining weight', which covered perceived integral messages delivered to patients by dietitians; and 'dietitian's approach' describing patient experiences of empathic and compassionate dietitians. Two overarching themes resulted from examining the connections and relationships between these dimensions: 'survival', a connection between eating and living; and 'support', describing the valued working partnership between dietitian and patient.

Conclusions Dimensions and themes overlapped with the qualitative literature on HNC patient experience of treatment. However, some themes, such as the empowerment of a message linking eating to survival, appeared unique to this study. Patients found this message to be delivered in a supportive manner that motivated change.
\end{abstract}

Keywords Head and neck cancer $\cdot$ Qualitative $\cdot$ Intervention $\cdot$ Malnutrition $\cdot$ Behaviour change

Electronic supplementary material The online version of this article (https://doi.org/10.1007/s00520-017-4029-5) contains supplementary material, which is available to authorized users.

Kristen McCarter

Kristen.McCarter@newcastle.edu.au

1 School of Medicine and Public Health, University of Newcastle, Callaghan, NSW 2308, Australia

2 School of Psychology, University of Newcastle, Callaghan, NSW 2308, Australia

3 Department of Radiation Oncology, Calvary Mater Newcastle Hospital, Waratah, NSW 2298, Australia

4 Centre for Dietetics Research, University of Queensland, St. Lucia, QLD 4067, Australia

\section{Background}

Patients with head and neck cancer (HNC) experience unique difficulties due to the malignancy, such as difficulty in eating, fatigue, loss of appetite and weight loss, and the treatment process can compound these problems [1]. The reported prevalence of malnutrition across all patients with cancer in Australia lies between 40 and $80 \%$ and patients with HNC are over-represented in this figure [2]. In addition to adverse treatment outcomes such as reduced resistance to the disease [3], poor nutritional status during treatment has been shown to be a strong predictor of mortality in $\mathrm{HNC}[4,5]$.

Multidisciplinary collaboration is important to the care of patients with diverse and complex needs such as the HNC population [6]. Oncology dietitians are considered a core 
member of this multidisciplinary team [7]. However, patients with HNC are often non-adherent with dietary advice [8]. Whilst there exists some qualitative literature exploring HNC patients' eating problems during treatment [3, 9], no qualitative studies exist that explore $\mathrm{HNC}$ patients' experience of dietetic consultations whilst undergoing treatment.

Most research focuses on the outcome of dietetic interventions rather than the patient experience of the working relationship with their dietitian, which may be crucial to understanding an intervention's effectiveness. In a recent randomised controlled trial, Britton et al. $[8,10]$ demonstrated that psychological strategies delivered by dietitians were successful in improving malnutrition and nutritional status in $\mathrm{HNC}$ patients. Given the efficacy of this intervention, understanding patients' experience of the intervention is a valuable addition to the literature. If dietitians are to be facilitators of nutritional behaviour change in the HNC population, then information regarding patient perceptions of the most effective components of intervention would be invaluable.

The present study explored experiences of HNC patients receiving a novel dietitian-delivered health behaviour intervention based on motivational interviewing (MI) and cognitive behavioural therapy (CBT) as part of a larger investigation examining the effect of this intervention on malnutrition in HNC patients undergoing radiotherapy (RT). Our overall aim was to provide patients the opportunity to share their experience of the intervention as an important component in considering its suitability for broader dissemination. As the intervention was newly developed, specifically, we wanted to explore the patient's working relationship with the dietitian, specific components of the EAT Intervention and suggestions for improving the intervention.

\section{Method}

This study is reported consistent with the Consolidated Criteria for Reporting Qualitative Research Checklist (COREQ) [11].

\section{Interviewer characteristics}

Author KB conducted the interviews with participants. KB is a clinical psychologist and research psychologist within the research team, experienced in conducting follow-up assessments.

\section{Relationship with participants and reflexivity}

No prior relationship was established with the interviewer (KB) and the participants prior to the commencement of the interviews. However, KB had previously rated audio recorded dietetic consultations for fidelity to the intervention delivered in the larger investigation. Participant knowledge of the interviewer was that she was a member of the EAT research team; no other interviewer characteristics were revealed, e.g. reasons and interests in the research topic. The authors of the current paper were involved in the overarching trial. The second coder (KM) was not known to the participants. As researchers, the coders made conscious efforts not to accept previous assumptions based on knowledge of the intervention content.

\section{Study design}

\section{Methodological orientation and theory}

Grounded theory [12] was the methodological orientation used to underpin the study. Due to the novel intervention employed in the original study, grounded theory was chosen to investigate the actualities in the real world [12] rather than other methodologies that may require pre-existing ideas or hypotheses. As explained by Strauss and Corbin [13], using grounded theory, researchers analyse and gather data simultaneously, allowing for formulation and exploration of provisional ideas regarding the participants' experiences as interviewing continues.

\section{Participants}

\section{Sampling}

An initial purposive sampling strategy was used and participants were drawn from a pool of cancer patients who had consented to take part in a larger investigation that evaluated the effectiveness of a dietitian-delivered health behaviour intervention to reduce malnutrition in patients with $\mathrm{HNC}$ undergoing RT: eating as treatment (EAT) $[8,10]$.

Participants were selected for the interviews on the basis of their consent to participate in future research (provided at commencement of the EAT study), being an intervention participant and their receipt of key components (described below) of the intervention (determined via a random sample of dietetic consultation audio recordings rated for fidelity to the intervention).

Patients' construction of the key messages around the importance of eating and maintaining weight, in addition to more general treatment related topics (e.g. side effects) during interviews, confirmed the decision to continue sampling from those that had received key components of the intervention.

\section{Method of approach}

Ethical approval was granted by the Hunter New England Human Research Ethics Committee (HREC) of Hunter New England Health (HREC/12/HNE/108; HNEHREC: 12/04/18/ 4.06). Only the primary researchers had access to the raw data. 
Information and consent forms were mailed to eligible participants asking if they were willing to participate in a tape recorded telephone interview with a research assistant. An option to withdraw from future studies related to the larger investigation was also included. The information form described that the aims of the project were to better understand the experience of patients who participated in the EAT dietetic intervention and also to obtain any feedback on how the intervention might be improved. A reply paid envelope was included for participants to return their signed consent form if they wished to participate. A follow-up telephone call was made to those who agreed to participate to arrange a convenient time to complete the telephone interview.

\section{Sample}

One hundred and fifty-six participants received the EAT Intervention in the overarching trial. A $20 \%$ sample of audio recorded dietetic sessions was rated for fidelity to the intervention [14]. Twenty-two participants were identified as eligible for the interviews based on these fidelity ratings. Two potential participants declined to participate by returning withdrawal forms. Ten potential participants did not return either consent or withdrawal forms. Ten potential participants initially agreed to be interviewed (one was subsequently unable to be contacted). A total of nine participants (all male) ranging in age from 53 to 75 years were interviewed (Table 1). The participants were drawn from each of the intervention sites. In accordance with grounded theory's constant comparative method [12], we interviewed participants and analysed and interpreted the data in an ongoing approach. We continued interviewing participants until we reached 'theoretical saturation'. This is what Glaser and Strauss (1967) referred to as the stage at which participant interviews no longer seem to be generating new information.

\section{The EAT Intervention}

Within the larger investigation, funded by Australia's National Health and Medical Research Council, four Australian radiotherapy (RT) departments that provide treatment for patients with $\mathrm{HNC}$ were recruited. Dietitians were trained, supervised and coached in the provision of the intervention known as EAT. The EAT Intervention incorporates $\mathrm{MI}$ and cognitive behavioural strategies [8, 10, 15] and was designed to increase the engagement of HNC patients with dietetic intervention, despite a range of barriers commonly encountered by this patient group; including local tumour effects, RT side effects (e.g. mucositis, dysgeusia, xerostomia, fatigue [1] and mental health problems [16]. Although the training was standardised, EAT is not a linearly structured intervention. Rather, the skills and principles can be flexibly integrated by dietitians into routine consultations.

A detailed description of the EAT Intervention is provided elsewhere [8]. Briefly, key elements included conducting a validated nutritional assessment (Patient-Generated Subjective Global Assessment; PG-SGA) [17]: the 'EAT to Live' conversation (whereby the dietitian would use MI to elicit the fundamental patient motivation of survival and link this to both the role of nutrition in RT and the current nutrition behaviours of the patient) and collaboratively developing (and subsequently reviewing) a written nutrition planner. MI principles and skills were used to guide delivery of all intervention elements. In the MI interactional style, clinicians are empathic, collaborative and elicit motivation for change from the patients themselves [18]. Dietitians were encouraged to integrate the intervention throughout usual practice from week one of radiotherapy to 12-week post radiotherapy, although it was expected that the EAT to Live conversation would occur (at a minimum) during week five of RT (when difficulties maintaining nutritional status were expected to peak).

Due to the emotional nature of the conversation raising discrepancy between a patient's current behaviour and the 'ideal' nutritional behaviour and the link made to survival, we were particularly interested in the EAT to Live conversation when interviewing patients.

\section{Setting}

The interviews were conducted via telephone and audio recorded. Only the interviewer and participant were present and recorded during the interview.

\section{Procedure}

Our telephone interviews, conducted over 7 weeks, were open ended and lasted an average of $16 \mathrm{~min}$ (range, 9-30 min). The interviews took place during August and September 2016, between 7 and 26 months after patients had completed the EAT Intervention (which ended 12 weeks post RT).

\section{Data collection}

The interviewer began the interviews by asking for general feedback about being part of the EAT study. The interviewer also asked about the patient's working relationship with the dietitian, specific components of the EAT Intervention and suggestions for improving the intervention. In general, the interviewer's approach was to use broad questions and reflection of content and feeling to encourage participants to elaborate. A semi-structured interview guide was used to guide initial interviews (supplementary file 1) to ensure that questions were asked in an open non-directive manner, allowing participants to 
Table 1 Participant

demographics $(N=9)$

\begin{tabular}{|c|c|c|c|}
\hline Demographics & Mean & SD & $\begin{array}{l}\text { Number } \\
(\%)\end{array}$ \\
\hline Age (years) & 61.3 & 6.5 & \\
\hline Male & & & $9(100)$ \\
\hline \multicolumn{4}{|l|}{ Tumour site } \\
\hline Nasopharynx & & & $1(10)$ \\
\hline Oropharynx & & & $7(80)$ \\
\hline Oral cavity & & & $1(10)$ \\
\hline Time since radiotherapy completion (months) & 17.9 & 7.7 & \\
\hline \multicolumn{4}{|l|}{ Tumour stage } \\
\hline I & & & $1(10)$ \\
\hline \multicolumn{4}{|l|}{ II } \\
\hline III & & & $1(10)$ \\
\hline IV & & & $7(80)$ \\
\hline Radiotherapy & & & $9(100)$ \\
\hline Surgery prior to radiotherapy & & & $2(20)$ \\
\hline Concurrent chemotherapy & & & $7(80)$ \\
\hline Prophylactic PEG & & & $2(20)$ \\
\hline Prophylactic NGT & & & - \\
\hline $\begin{array}{l}\text { Percentage weight loss (at } 3 \text { months post radiotherapy as a percentage of first week } \\
\text { of radiotherapy weight) }\end{array}$ & 9.0 & 4.8 & \\
\hline
\end{tabular}

speak freely about their experiences. Following initial open coding, the interviewer moved back and forth between data collection and analysis with questions guided by our developing coding.

Audio recordings of the telephone interviews were sent to an online professional service for transcription. The transcriptions were checked by the data coders (KB and KM).

\section{Data analysis}

The data was analysed using grounded theory $[12,13]$. As such, our themes were not identified in advance but derived from the data. Consequently, we employed a process of open and axial coding and then final selective coding to organise the data. In conducting the coding, two researchers (KB and KM) were given copies of all transcripts for analysis. Each level of coding generated a discussion until consensus was reached.

Open coding is a preliminary process of generating categories from the data in order to group it into larger conceptual categories. Axial coding involves greater interpretation and the various open coding categories are incorporated to form broader categories, whereby themes begin to emerge. Selective coding involves searching for the meaning, connection or richer themes among the axial codes.

Data was managed and analysis conducted using QSR NVivo version 11.

\section{Results}

\section{Initial analysis}

Open coding revealed the categories 'dietitian helpful', 'trouble eating' and 'feeling comfortable'. Using axial coding we developed these categories by identifying properties and dimensions along which participants described their experience of the EAT Intervention. In these, participants highlighted the following: (1) information, (2) challenges of treatment experience, (3) key messages and (4) dietitian's approach. These four dimensions are presented below.

\section{Information}

The information that patients received was an important part of their overall experience. Some participants described that the dietitians not only offered them 'options' in what they could eat, but also went further to explain why particular foods were needed, for example 'explaining the different food groups which gave me the proteins or whichever I needed', which made things 'clearer'. However, other patients felt that the information regarding food options was either lacking, such as not being informed about nutritional supplement options 'until I was just about to go on to the nasal feed' or 'very general', and would have appreciated advice that was tailored to take into consideration their individual side effects or medical or economic circumstances. 'There was no real recipes. 
You know, there was no try doing this, or this sort of food'. In terms of information related to side effects, patients felt that they were well informed as to what they could expect and how 'they went just that bit further with explaining'.

\section{Challenges of treatment experience}

Side effects All patients interviewed described the side effects associated with HNC and RT. This included descriptions such as sore throat, ulcers, taste and smell changes, lack of saliva and swallowing problems. Many of the patients described having more than one issue that affected their ability to eat. The patients noted the length of time the side effects continued was 'much longer than I'd initially expected', particularly the fact that side effects often get worse and persist after treatment. 'I thought, you know, two weeks from now I'll be up and about doing what I always used to do. But in fact at eight weeks I still had a very sore throat and I think it was ten or 12 weeks before I had definitely turned the corner and I could see that I was getting better'.

Finding food to eat A number of patients described that the biggest hurdle was finding recipes and foods to eat. They found it 'frustrating' that 'there's not a lot of options out there'.

Additional support pre- or post treatment Many of the patients identified that linking with a dietitian earlier in their treatment trajectory would have been helpful, particularly in regard to receiving the key message of the importance of maintaining or even gaining weight prior to treatment.

Several of the participants described that they would have liked more follow-up support from either the hospital or dietetics department after their treatment finished. One rural participant described the challenges in accessing support following treatment in regards to dental care 'I think that's the only part, that there should be a complete follow up all the time on cancer patients that have had to take their teeth out'. In addition to practical support with maintaining oral health, some participants desired more information for the survivorship period, 'you know, a years' time expect this, in two years' time expect this'.

\section{Key messages: importance of eating and maintaining weight}

The participants indicated that they were aware of the importance of food intake and nutrition, '...your body actually lets you know that it's true. You're in no doubt about whether it's true or not because of the demands from your body are just so great'. 'I had to keep the nutrition in me to keep my strength up, to help with, you know, like fighting the fight against the fight if you know what I mean?' The participants also identified the significance of maintaining weight; 'I kept thinking about that the whole time'. '...the key message about maintaining weight, for me, was an important barometer, and there is [a] focus on weighing you when you come in for your weekly, weekly I think it was, you know, monitoring that. But just knowing that's a guide for how you're going, it was an obvious one, but nonetheless you could self-monitor that at home and you could adjust your intake accordingly'.

\section{Dietitian's approach}

Patients identified factors in the overall approach or style of the dietitian which affected their experience.

Empathy The majority of participants reported that their sessions with the dietitian were not only 'helpful' but that they felt 'comfortable' with the dietitians, as they were 'understanding'; 'we're all in the same boat there somehow'. Conversely, some doubted that dietitians would know 'what it's like from a patients' point of view' because 'nobody really knows what it's like unless you've been through it'. This suggested that patients felt that it was important that the dietitians empathised with them and that this was element that contributed to the 'good relationship' and between the two described by those who found their dietitians empathic; '... engagement was strong'.

Compassion Compassion was valued by patients in addition to practical information. Participants felt that an important part of the dietitians' role was to be 'positive' and 'reassuring, you know like, "You'll make it through this, and you'll get through it all"', 'But without being too abrupt they were quite gentle in the way they were saying it'.

In our final coding process, we examined the four dimensions identified during axial coding and looked for relationships or connections among the categories identified in open coding as well as in participants' transcripts that might serve to generate a more meaningful reflection of how they experienced the intervention and their dietitians. This selective coding resulted in the identification of two encompassing themes that reflected what participants were telling us about their experience of the intervention. Firstly, participants appeared to be saying that the challenges of the treatment experience resulted in changes to the meaning of food and subsequently a focus on survival as a key coping strategy. Secondly, it was important to receive proper and sufficient information from a caring and empathic dietitian to feel adequately supported.

Survival Being aware of the necessity to eat to manage the treatment gave rise to a connection between eating and living. Survival became a focus for the patients, 'you know you have to eat to survive', '...you've got no tastebuds, or nothing tastes of anything, so and you just know that you've got to put something in your mouth to stay alive'. Patients described 
their perseverance, 'Like I say, I was still eating well despite the sore throat, the soreness of it, which increased... But I kept eating'. It seemed that dietitians had a key role to play in elevating eating to a strategy that could mean the difference between life and death; '...the dietitians make you extremely aware of it and leave no doubt', 'that link was very clear', 'Yeah, well see I wasn't conscious of that until they did bring that up and say, "Look, you know, if you haven't maintained some weight, and when you finish the treatment, it can cause you to... you can die, you know, post treatment." And I thought, oh OK'. Interestingly, whilst the connection drawn between nutrition and survival was associated with a level of discomfort, 'a bit of fear and anxiety', the link also empowered them; 'I think that was reassuring, you know, keep an eye on that, keep on top of it', 'knowing that you could maintain the weight was also a bonus, a plus, it's good to know that you had control over something, that was a positive feeling'.

Support Inherent in the participants' interviews about their experience of the intervention was the notion that they wanted information delivered by a supportive professional. Participants valued a competent professional with expertise, 'I felt as though they knew precisely what they were supposed to do and what you needed to know about it'. Whilst receiving helpful information and advice was appreciated, a supportive partnership was no doubt an important part of valued working relationships between the patient and their dietitian. 'Yeah, they sort of help you mentally through it', 'You know like they become nearly your friends'. 'So it was like the whole program is actually just to try to help you get along and sort of get going again. So my experience with the dietitians, I find them very useful, they're an important part of the program'.

\section{Discussion}

Analysis of conducted interviews identified key messages and common factors that affected patients' experience and resulted in four dimensions and two overall themes. A major finding of the present study was the theme that patients linked eating with survival. The EAT Intervention emphasises that maintaining adequate nutrition during RT is an integral part of cancer treatment and not merely an adjunct to survival [8]. The EAT to Live conversation employed by the dietitians elicits patients' reasons for having RT. Ultimately, a core reason for undergoing the rigours and sometimes severe side effects of RT includes some elements of wanting to live. In offering an invitation to explain the correlation between malnutrition during RT and poorer outcomes, the dietitian is then able to deploy variance by inviting the patient to reflect on their continued RT attendance and their concurrent nutritional behaviours. These current behaviours may not be consistent with the likelihood of meeting the core goal of living. The dietitian endeavours to convert the motivation elicited into concrete dietary behavioural changes.

Prior qualitative research exploring eating problems for patients with $\mathrm{HNC}$ during RT has found that patients are aware of the necessity of eating to manage the treatment and illness, but this leads to frustration and feelings of uncertainty about whether the treatment was worth it [19]. Additionally, patients with HNC have previously been reported to be noncompliant with dietetic advice [8]. In the current study, the importance of eating and maintaining weight to ensure survival despite the challenges of treatment was shown to be a central message delivered by dietitians. Further, patients described not just receiving this message but implementing and even feeling empowered by it.

The other key themes of the current study are the description and appreciation of empathic, compassionate and ultimately supportive dietitians. That the patients perceived dietitians as supportive indicates that the dietitians were able to 'deploy discrepancy' [18] (between not eating and wanting to survive) in the EAT Intervention in a motivating and compassionate manner. Deploying variance requires a good rapport and genuineness for it not to seem accusatory and confrontational [8]. This finding supports existing research that indicates greater empathy in dietetic consultations results in improved patient satisfaction and treatment adherence [20, 21]. The first principle of the EAT Intervention refers to the MI interactional style in which clinicians are empathic, collaborative and elicit motivation for change from the patients themselves $[8,18]$. The extent to which dietitians employed the principles of the intervention is reported elsewhere [14]. The findings of the current study indicate that this interactional style was valued by the participants and should be incorporated into dietetic consultations.

Whilst the four dimensions originally identified in the coding process are represented in the two themes described above, there are a number of noteworthy points to discuss from the original categories. The participants' comments in regard to information suggest that it is important for the dietitian to understand the patient's needs and wants and to tailor information and support based on this. This finding aligns with previous qualitative research of patient experiences of dietetic consultations in other health settings that has found patients value explanation of information and advice and adaptation of advice to individual circumstances [20]. For those participants who felt the information given to them by their dietitians was lacking or too generic, it may be that there was a tension between what the patient identified as important and the dietitian's agenda. This divergence has been previously identified by MacLellan and Berenbaum [22] in their study that describes the difficulty in balancing client-centred approaches and dietitian determination of patients' needs. 
The eating problems and side effects of treatment described by participants support previous research and knowledge of the unique challenges faced by those with HNC [1]. The desire expressed by patients for earlier intervention, greater follow-up support and specifically further information on the impact of their treatment, for example the length of time side effects of treatment persist, is also consistent with previous research. In a study aimed at determining how satisfied $\mathrm{HNC}$ patients are with the information they receive pre-treatment, Llewellyn et al. [23] reported key areas of improvement identified by patients. These included long-term effects of treatment on ability to work, physical functioning and quality of life.

Further, a qualitative study by McQuestion et al. [9] exploring physical, social and emotional loss for patients having received radiation treatment for $\mathrm{HNC}$ found that patients need to be better prepared for the post treatment phase and the slow recovery. This was particularly relevant to realistic expectations about the pace and timeframe of recovery. Patients' information needs differ, and when adequate information is not provided, they may be ill prepared for certain aspects of treatment and recovery. Unmet informational needs and low satisfaction with information provision has been associated with adverse patient outcomes including poorer health-related quality of life $[24,25]$. Consequently, it is important to address patients' informational needs prior to treatment.

This finding might also be explained by dietetic patients' desire for dietitians to understand the individuality of the patient and to identify the outcome they want from the consultation and base information on this [20]. One specific component of the EAT Intervention included training dietitians in collaborative agenda setting. However, analyses revealed that training did not significantly improve the application of this skill [14]. It may be that the desire expressed by some patients in the current study for tailored information is a result of this lack of improvement. It may be useful to place greater emphasis on strengthening this skill during training in future implementations of the intervention.

The EAT Intervention sought to address the inherent difficulties in intervening with the HNC population by providing dietitians with training, skills and knowledge to deal with this difficult and often overlooked group [8, 14]. The trial demonstrated significant and clinically meaningful benefits of psychological strategies, delivered by dietitians, to improve malnutrition in HNC patients $[10,14,15]$. This qualitative investigation further strengthens the findings of the EAT study by demonstrating that dietitians are well placed to play a key role in HNC patients' perceptions of the role of nutrition in surviving treatment, and this message can be delivered to empower and motivate patients to action. Given the success of the EAT intervention in improving nutrition in $\mathrm{HNC}$ patients receiving radiotherapy [10], coupled with the findings of the current study that intervention patients linked eating with survival and perceived dietitians as supportive, this intervention should be considered for use in radiotherapy departments in which malnutrition in $\mathrm{HNC}$ patients is a problem. Future implementation of the intervention should include efforts for earlier intervention, more extensive follow-up support and greater information provision on the potential impacts of treatment.

In regard to treatment outside of future implementation of the EAT intervention, our findings add to the evidence for complex needs of this patient population [1-5], specifically prior to and following radiotherapy treatment. This is also consistent with evidence that patients continue to report unmet supportive care needs years after their cancer diagnosis [26] and highlight the need for greater multidisciplinary team care [27-29].

\section{Limitations}

The findings are representative of the experience of patients according to their recall. These recollections may be different from what might have been the experience of participants at the time they experienced the EAT Intervention, especially when discussing the impact of an intervention they received between 7 to 26 months prior. The accounts of nine patients working with six dietitians are representative of our intervention sample but may not reflect the variety of possible experiences that patients with head and neck cancer may have during treatment and therefore restricts generalizability of the findings. In regard to trustworthiness, the modality of the interviews, i.e. telephone interviews, may have contributed to their relatively short length. Telephone interviews were used due to the participants being located across Australia. Despite the decision to cease interviews based on data saturation, it may be that further information could be gathered in face to face interviews or using focus group methodologies. However, the results of this study represent a depth of understanding that adds to the main findings of the EAT trial that may not have otherwise been uncovered with alternative methods. Given the unique difficulties associated with treatment for head and neck cancer, understanding patient experience and perspectives is vital to informing the development of successful interventions.

\section{Conclusions}

Dietitians can be trained to deliver a health behaviour change intervention, EAT, to help prevent malnutrition during RT for HNC. This intervention appears to assist patients to link eating with survival and was perceived as being empathically delivered in a supportive context. Furthermore, this approach even facilitates a feeling of control and motivation in the difficult $\mathrm{HNC}$ treatment journey.

Acknowledgements The authors would like to thank the study participants for their invaluable contribution. 
Funding information This work was supported by a Calvary Mater Newcastle James Lawrie Research Fund grant. The EAT trial was supported by the National Health and Medical Research Council (APP1021018; 2011/3654).

Compliance with ethical standards Ethical approval was granted by the Hunter New England Human Research Ethics Committee (HREC) of Hunter New England Health (HREC/12/HNE/108; HNEHREC: 12/04/ 18/4.06).

Conflict of interest The authors declare that they have no conflict of interest.

Research involving human participants and/or animals All procedures performed in studies involving human participants were in accordance with the ethical standards of the institutional and/or national research committee and with the 1964 Helsinki Declaration and its later amendments or comparable ethical standards.

Informed consent Informed consent was obtained from all individual participants included in the study.

Open Access This article is distributed under the terms of the Creative Commons Attribution-NonCommercial 4.0 International License (http:// creativecommons.org/licenses/by-nc/4.0/), which permits any noncommercial use, distribution, and reproduction in any medium, provided you give appropriate credit to the original author(s) and the source, provide a link to the Creative Commons license, and indicate if changes were made.

\section{References}

1. Lees J (1999) Incidence of weight loss in head and neck cancer patients on commencing radiotherapy treatment at a regional oncology centre. Eur J Cancer Care 8(3):133-136. https://doi.org/10. 1046/j.1365-2354.1999.00156.x

2. Kruizenga HM, Wierdsma NJ, van Bokhorst MA, Hollander H, Jonkers-Schuitema C, Van Der Heijden E et al (2003) Screening of nutritional status in The Netherlands. Clin Nutr 22(2):147-152. https://doi.org/10.1054/clnu.2002.0611

3. Larsson M, Hedelin B, Johansson I, Athlin E (2005) Eating problems and weight loss for patients with head and neck cancer: a chart review from diagnosis until one year after treatment. Cancer Nurs 28(6): 425-435. https://doi.org/10.1097/00002820-200511000-00004

4. van Leeuwen PA, Kuik DJ, Klop WMC, Sauerwein HP, Snow GB, Quak JJ (1999) The impact of nutritional status on the prognoses of patients with advanced head and neck cancer. Cancer 86(3):519-527

5. Britton B, Baker A, Clover K, McElduff P, Wratten C, Carter G (2016) Heads up: a pilot trial of a psychological intervention to improve nutrition in head and neck cancer patients undergoing radiotherapy. Eur J Cancer Care 26(4). https://doi.org/10.1111/ecc.12502

6. Harris JR, Lau H, Surgeoner BV, Chua N, Dobrovolsky W, Dort JC, Kalaydjian E, Nesbitt M, Scrimger RA, Seikaly H, Skarsgard D, Webster MA, Members of the Alberta Provincial Head and Neck Tumour Team (2014) Health care delivery for head-and-neck cancer patients in Alberta: a practice guideline. Curr Oncol 21(5): e704-ee14. https://doi.org/10.3747/co.21.1980.

7. National Institute for Health and Clinical Excellence (NICE) (2004) Guidance on cancer services: improving outcomes in head and neck cancers - the manual. NICE, London
8. Britton B, McCarter K, Baker A, Wolfenden L, Wratten C, Bauer J, Beck A, McElduff P, Halpin S, Carter G (2015) Eating as treatment (EAT) study protocol: a stepped-wedge, randomised controlled trial of a health behaviour change intervention provided by dietitians to improve nutrition in patients with head and neck cancer undergoing radiotherapy. BMJ Open 5(7):e008921. https://doi.org/10.1136/ bmjopen-2015-008921

9. McQuestion M, Fitch M, Howell D (2011) The changed meaning of food: physical, social and emotional loss for patients having received radiation treatment for head and neck cancer. Eur J Oncol Nurs 15(2): 145-151. https://doi.org/10.1016/j.ejon.2010.07.006

10. Britton B, Baker AL, Wolfenden L, Wratten C, Bauer J, Beck AK et al (2017) Eating As Treatment (EAT): a stepped-wedge, randomised controlled trial of a health behaviour change intervention provided by dietitians to improve nutrition in patients with head and neck cancer undergoing radiotherapy. Manuscript submitted for publication

11. Tong A, Sainsbury P, Craig J (2007) Consolidated criteria for reporting qualitative research (COREQ): a 32-item checklist for interviews and focus groups. Int J Qual Health Care 19(6):349 357. https://doi.org/10.1093/intqhe/mzm042

12. Glaser BSA (1967) The discovery of grounded theory. Aldine Publishing, Chicago, IL

13. Strauss A, Corbin J (1990) Basics of qualitative research. Sage, Newbury Park

14. Beck AK, Britton B, Baker AL, Wratten C, Bauer J, Wolfenden L et al (2017) 'EAT': eating as treatment: training head and neck cancer dietitians in a health behaviour change intervention. Manuscript submitted for publication.

15. McCarter K, Baker AL, Britton B, Beck AK, Carter G, Bauer J et al Effectiveness of clinical practice change strategies in improving dietitian care for head and neck cancer patients according to evidence based clinical guidelines: a stepped wedge randomised controlled trial. 2017. Transl Behav Med. https://doi.org/10.1093/tbm/ibx016

16. Linden W, Vodermaier A, MacKenzie R, Greig D Anxiety and depression after cancer diagnosis: prevalence rates by cancer type, gender, and age. J Affect Disord 141(2):343-351. https://doi.org/ 10.1016/j.jad.2012.03.025

17. Bauer J, Capra S, Ferguson M (2002) Use of the scored patientgenerated subjective global assessment (PG-SGA) as a nutrition assessment tool in patients with cancer. Eur J Clin Nutr 56(8): 779-785. https://doi.org/10.1038/sj.ejcn.1601412

18. Miller WR, Rollnick S (2012) Motivational interviewing: helping people change. Guilford Press

19. Larsson M, Hedelin B, Athlin E (2003) Lived experiences of eating problems for patients with head and neck cancer during radiotherapy. J Clin Nurs 12(4):562-570. https://doi.org/10.1046/j.13652702.2003.00751.x

20. Hancock RE, Bonner G, Hollingdale R, Madden AM (2012) 'If you listen to me properly, I feel good': a qualitative examination of patient experiences of dietetic consultations. J Hum Nutr Diet 25(3):275-284. https://doi.org/10.1111/j.1365-277X.2012.01244.x

21. Goodchild CE, Skinner TC, Parkin T (2005) The value of empathy in dietetic consultations. A pilot study to investigate its effect on satisfaction, autonomy and agreement. J Hum Nutr Diet 18(3):181185. https://doi.org/10.1111/j.1365-277X.2005.00606.x

22. MacLellan D, Berenbaum S (2007) Canadian dietitians' understanding of the client-centered approach to nutrition counseling. J Am Diet Assoc 107(8):1414-1417. https://doi.org/10.1016/j.jada. 2007.05.018

23. Llewellyn CD, McGurk M, Weinman J (2006) How satisfied are head and neck cancer (HNC) patients with the information they receive pre-treatment? Results from the satisfaction with cancer information profile (SCIP). Oral Oncol 42(7):726-734. https://doi. org/10.1016/j.oraloncology.2005.11.013. 
24. Edwards D (1998) Head and neck cancer services: views of patients, their families and professionals. Br J Oral Maxillofac Surg 36(2):99-102. https://doi.org/10.1016/S0266-4356(98)90175-9

25. Mesters I, Van den Borne B, De Boer M, Pruyn J (2001) Measuring information needs among cancer patients. Patient Educ Couns 43(3):255-264. https://doi.org/10.1016/S0738-3991(00)00166-X

26. McDowell ME, Occhipinti S, Ferguson M, Dunn J, Chambers SK (2010) Predictors of change in unmet supportive care needs in cancer. Psycho-Oncology 19(5):508-516. https://doi.org/10.1002/pon.1604

27. Wiederholt PA, Connor NP, Hartig GK, Harari PM (2007) Bridging gaps in multidisciplinary head and neck cancer care: nursing coordination and case management. Int J Radiat Oncol Biol Phys 69(2, Supplement):S88-S91

28. Larsson M, Hedelin B, Athlin EA (2007) Supportive nursing care clinic: conceptions of patients with head and neck cancer. Eur J Oncol Nurs 11(1):49-59. https://doi.org/10.1016/j.ejon.2006.04.033

29. Wells M, Donnan PT, Sharp L, Ackland C, Fletcher J, Dewar JA (2008) A study to evaluate nurse-led on-treatment review for patients undergoing radiotherapy for head and neck cancer. J Clin Nurs 17(11):1428-1439 